\title{
Reflection and transmission of microwaves in reinforced concrete specimens irradiated by modified antipodal Vivaldi antenna
}

\section{Zahra Esmati ${ }^{1}$ and Mahdi Moosazadeh ${ }^{1}$}

${ }^{1}$ Center for Infrastructure Engineering, School of Computing, Engineering and Mathematics, Western Sydney University, Penrith NSW 2751, Australia

Email: z.esmati@westernsydney.edu.au

\begin{abstract}
In this letter the reflection and transmission properties of reinforced concrete specimens irradiated by a modified, high-gain, ultra-wideband antipodal Vivaldi antenna (MAVA) are investigated numerically and experimentally. First, the influence of the interfaces between free space and concrete, and a reinforced bar (rebar) cell embedded in concrete on the reflection property is investigated at frequency range from 0.65-3.5 GHz. Second, the transmission property of the reinforced concrete specimens is investigated at $2.45 \mathrm{GHz}$ using a small resonant microstrip patch antenna embedded in concrete at the location of a rebar cell with different values of cell parameter. It is shown that the reflection from the free space-concrete interface of specimens with and without rebars provides the most critical effect on the reflection and transmission properties of the specimens. It is also shown that maximum transmission can be achieved when the patch antenna is located in the centre of the rebar cell. The measured results are in good agreement with the numerical results. The results of this letter can be used for nondestructive testing of reinforced concrete specimens and for wireless power transmission to sensors embedded in reinforced concrete.
\end{abstract}

Key words: antipodal Vivaldi antenna; reinforced concrete; non-destructive testing and Ebaduatton author manuscript accepted for publication and has undergone full peer review but has not been through the copyediting, typesetting, pagination and proofreading process, which may lead to differences between this version and the Version of Record. Please cite this article as doi: $10.1002 /$ mop.31307 


\section{INTRODUCTION}

Reinforced concrete is widely used in infrastructure systems such as building, bridges, roads and dams. One of the main features of a reinforced concrete structure is a grid of cells formed by reinforcing bars (rebars) embedded in concrete to increase its strength and serviceability. The influences of the reinforced concrete structure on propagation of electromagnetic waves have been investigated for decades. The main frequency region of interest includes microwave frequency range $(300 \mathrm{MHz}-30 \mathrm{GHz})$ because signals at these frequency, in particular at the lower range can penetrate and propagate in concrete, and transmit through concrete specimens, walls, etc. These investigations are mostly related to communication applications and they include a fundamental study of scattering and resonant phenomena in reinforced concrete [1-2]. Nondestructive testing (NDT) of reinforced concrete specimens and structural health monitoring (SHM) of infrastructure systems are other applications of microwaves where the influence of the reinforced concrete structure plays an important role. The NDT uses reflection and/or transmission properties of concrete for its characterization, and the detection and evaluation of flaws in its members [3]. Monitoring the performance of the infrastructure systems and evaluation of its health state using sensing techniques are the main purposes of SHM. Microwave techniques have been used as sensing as well as wireless communication techniques for SHM. Antennas are the main components of the microwave techniques used in these applications. For instance, open-ended waveguide antennas and lens antennas have been widely used for NDT of composite materials [4]. In [5], the effects of reinforced bars on coupling between two microstrip patch antennas were investigated numerically; however, experimental verification of these simulated results was not provided. Two microstrip patch antennas operating at $2.5 \mathrm{GHz}$ were used in [6] to investigate the transmission property of concrete specimens numerically and 
experimentally. The results showed that a $-30-\mathrm{dB}$ coupling can be achieved when an external patch antenna is located at depth of $100 \mathrm{~mm}$ in concrete. In addition, in [7] the return loss and transmission characteristics of dipole inside a sample of a concrete bridge pier were investigated. It was found that when dipole antennas are located inside concrete, antennas had desired return loss characteristics. However, dipole antennas did not work properly in reinforced concrete samples because of their relatively large length.

Recently, antipodal Vivaldi antennas (AVAs) have been developed and applied for NDT of construction materials and composite structures. These antennas have advantages such as wide/ultra-wide frequency bands, relatively small dimensions and high gain. For instance, the AVA for both sensing and data transmission was used for pipe telemetry applications in [8]. A novel technique for through-the-wall imaging using AVA aimed at the detection of stationary target was presented in [9]. In [10-14] AVAs have been developed and used for detection of defects such as voids and cracks in construction materials and concrete structures.

Research performed by the authors of this letter has been focused on NDT of construction materials and structural health monitoring of infrastructures using continuous wave microwave technique with the modified AVA (MAVA). Construction materials include concrete, metal, polymers, glass, wood and ceramic, which have different electromagnetic properties and require different operating frequency ranges. Recently, a relatively simple AVA has been proposed in [15] for an investigation into the feasibility of the detection of air gap inside a concrete block at a relatively low and narrow frequency range $(0.65-2.6 \mathrm{GHz})$.

In this letter for the first time, reflection and transmission of microwaves in reinforced concrete specimens irradiated by MAVA operating at a relatively wide frequency range $(0.65-3.5$ $\mathrm{GHz}$ ) have been investigated numerically and experimentally using a one-unit-cell approach. 
This allowed us to simplify the preparation of specimens and measurements as well as to compare our results with published ones related to the NDT applications. The reflection property is investigated at frequency range of $0.65-3.5 \mathrm{GHz}$ whilst the transmission property of the reinforced concrete specimens is investigated at $2.45 \mathrm{GHz}$ using a small resonant microstrip patch antenna embedded in concrete at the location of rebar cell with different values of cell parameter. Modelling and numerical study are performed with Computer Simulation Technology (CST) Microwave Studio by simulating S-parameters as measures of the reflection and transmission properties, far-field radiation patterns in free space and electrical field intensity distribution in the specimens. Experimental measurements are conducted with an Agilent performance network analyser (PNA). Section 2 briefly presents the design and performance of the MAVA. Section 3 deals with results and discussions of the reflection property of the reinforced concrete specimens irradiated by MAVA at frequency range of 0.65-3.5 GHz. Section 4 presents the results pertaining to the transmission property of the specimens at $2.45 \mathrm{GHz}$ between MAVA and small resonant microstrip patch antenna. Finally, concluding remarks are presented in Section 5.

\section{DESIGN AND PERFORMANCE OF THE MODIFIED ANTIPODAL VIVALDI}

\section{ANTENNA IN FREE SPACE}

Schematic of the MAVA is shown in Fig. 1(a). The proposed antenna is printed on Rogers 5880 with relative dielectric constant of 2.2, loss tangent of 0.009 , and thickness of $0.508 \mathrm{~mm}$. As can be seen from Fig. 1(a) two symmetrical radiators are located on two opposite sides of the substrate. The shape and dimensions of the inner and outer edge of the elliptical radiation flares are designed using the approach described in [15]. Optimal dimensions of the antenna have been 
determined using extensive simulation with “open (add space)" boundary conditions in CST and are as follows: $L_{\mathrm{sub}}=202 \mathrm{~mm}, W_{\mathrm{sub}}=171 \mathrm{~mm}, W_{\mathrm{f}}=1.53 \mathrm{~mm}, L_{\mathrm{f}}=62 \mathrm{~mm}, R=48 \mathrm{~mm}, a_{1}=50$. $765 \mathrm{~mm}, b_{1}=290 \mathrm{~mm}, a_{2}=49.235 \mathrm{~mm}, b_{2}=40 \mathrm{~mm}, a_{3}=35 \mathrm{~mm}, b_{3}=49 \mathrm{~mm}$, and $L_{1}=32 \mathrm{~mm}$.

The optimized antenna was fabricated and a photograph of the fabricated antenna is shown in Fig. 2(b). It should be mentioned that a coaxial connector and a cable were connected to the fabricated MAVA for measurement of its performance while the model of MAVA in CST was created without connector and cable. The measured and simulated reflection property (as $S_{11}$ in $\mathrm{dB}$ ) of the proposed antenna in free space is shown in Fig. 1(b). It can be seen from Fig. 1(b) that the measured and simulated $S_{11}$ demonstrate a similar trend and behaviour and the differences may be due to the fabrication error and the influence of coaxial connector and cable. The farfield radiation patterns of the proposed antenna at three frequencies are depicted in Fig. 1(c). It can be seen in Fig. 1(c) that the antenna exhibits end-fire characteristics at three frequencies. To highlight a relatively high gain at low frequencies and small dimensions of the proposed antenna, Table 1 shows its maximum gain along with maximum gain of the referenced antennas at three different frequencies. It can be seen from Table 1 that although some of the referenced antennas (e.g., in [9] and [16]) operates at $0.9 \mathrm{GHz}$, the gain value at this frequency is low in comparison with the proposed antenna. For instance, in [16] the antenna operated from $0.4 \mathrm{GHz}$ to $9.8 \mathrm{GHz}$; however, the gain of antenna is zero at $0.9 \mathrm{GHz}$ while this antenna has larger dimensions compared to the proposed antenna.

\section{REFLECTION PROPERTY OF REINFORCED CONCRETE SPECIMENS}

In order to investigate the influence of rebars on the reflection property of reinforced concrete specimens irradiated by MAVA, extensive simulations and measurements with the fabricated 
MAVA and reinforced concrete specimens were conducted. The most critical simulated and measured results at the frequency range from $0.6-3.5 \mathrm{GHz}$ and at selected single frequencies are presented in this section. Fig. 2(a) shows the model of the MAVA with a rectangular reinforced concrete specimen and the distance between them, $d_{1}$. The specimen has dimensions of $250 \mathrm{~mm}$ by $250 \mathrm{~mm}$ by $382 \mathrm{~mm}$, dielectric constant of 4.5 , and loss tangent of dry concrete of 0.0111 . A rebar cell with parameter $g$ is embedded in concrete so that the distance between the top (bottom) surface of specimen and the center of lower two rebars, $d_{2}\left(d_{3}\right)$, and upper two rebars, $d_{4}$, are 132 $\mathrm{mm}(250 \mathrm{~mm})$ and $100 \mathrm{~mm}$, respectively. Each rebar has a diameter of $16 \mathrm{~mm}$ and was made of Steel-1008 (conductivity of $7.69 \times 106 \mathrm{~S} / \mathrm{m}$ ). The model with a unit cell was created using general purpose frequency domain solver open boundaries realized by a Floquet port. In addition, mesh enhancement methods were used to improve the simulation accuracy. Fig. 2(b) shows the measurement setup. The specimen used in this setup consists of a 100-mm thick concrete slab with dimensions of $250 \mathrm{~mm}$ by $250 \mathrm{~mm}$, and a $250-\mathrm{mm}$ concrete cubic block. The rebar cell consisting of four 16-mm diameter rebars was located between the concrete block and slab as shown in Figs. 2(b-c). The rest of 16-mm gap between the slab and the block was filled by pieces of plasterboard with a dielectric property close to dielectric property of concrete block and slab used in this investigation. $S_{11}$ of the MAVA in free space was measured using the PNA calibrated with Agilent Electronic Calibration Module (ECal).

Simulated and measured $S_{11}$ at three distances between the antenna and the concrete specimens with and without rebar cell at $g=110 \mathrm{~mm}$ are shown in Fig. 3. It should be noted that the simulated results were obtained using 1001 frequency points while the number of frequency points during the measurement was 201 . First, the simulated $S_{11}$ for the antenna with the concrete specimen without rebars (c.f. Fig. 3(a)) and in free space (c.f. Fig. 1(b)) is presented. The 
comparison shows the following: 1) the presence of concrete specimen increases reflection at most frequencies and creates multiple ripples and resonant responses that can be attributed to the reflection from the specimen boundaries, in particular from the top surface irradiated by the MAVA; 2) $S_{11}$ of the concrete specimen at some frequencies corresponding to the resonant dips is lower than in free space; for instance, the reflection reduces significantly from $0.6-0.8 \mathrm{GHz}$ at $d_{1}=0$ and from 1.4-1.7 GHz at $d_{1}=0$ and $100 \mathrm{~mm}$. Second, we considered the influence of rebars on the simulated $S_{11}$. The main observation is that embedding the rebar cell leads to changes of magnitude of the dips and there are small shifts in the frequency corresponding to the dips at all d1 values while no new dips occur. The most significant changes can be seen at $d_{1}=0$. Overall, the simulated results show that the reflection from the boundaries of specimen plays a dominant role in relative (to free space) changes of the $S_{11}$. The measured results demonstrate a similar behavior of the simulated $S_{11}$. However, it is worth mentioning that embedding the rebars increases the number of dips at $d_{1}=50 \mathrm{~mm}$ and $100 \mathrm{~mm}$, respectively, compared with the case at $d_{1}=0$. These peaks occurred due to the influence of the rebars. In general, the results shown in Fig. 3 demonstrate that one of the main features of reinforced concrete specimens irradiated by the MAVA, is the indication of multiple ripples and the resonant responses at the reflection spectrum. Therefore, one of the advantages of wideband antennas, such as MAVA, is the opportunity to select a desired single frequency for a certain application.

Simulation and analysis of electrical field intensity (referred to as E-field) distributions in MAVA and the specimens have been performed at several single frequencies over the operating frequency range at different $d_{1}$ distances to further investigation of the reflection as well as propagation of microwaves in the specimens irradiated by the MAVA. Fig. 4 shows selected results of the E-field distributions obtained at $d_{1}=50 \mathrm{~mm}$ and at a few single frequencies at the 
same scale. At all selected frequencies the antenna radiates toward the specimen and the electromagnetic radiation is partly reflected from air-to-concrete interface, partly transmits into the specimen and propagates through the sample. The propagation of the electromagnetic waves in the sample is a complex function of the operating frequency, polarization of the antenna, and the presence of rebar cell. Moreover, the analysis of E-field distributions at low frequencies clearly shows that the electromagnetic waves are almost blocked by rebar cell at $0.7 \mathrm{GHz}$ as shown in Fig. 4(a). At this frequency, the influence of the rebar cell on the reflection property of the specimen is significant, i.e., it leads to an appearance of the resonant peak at the simulated and measured reflection spectra as shown in Fig. 3(b). This happened because parameter $g$ is equal half electrical wavelength $(110 \mathrm{~mm})$ at this frequency and as a result, microwaves at this frequency are significantly shielded by the rebar cell similar to the case described in [7]. Another important observation can be made from Fig. 4(b) at frequency of $2.02 \mathrm{GHz}$. It can be seen that microwaves propagate through the rebar cell and the specimen (c.f. Fig. 4), and there are no dips and the indication of the influence of the rebar cell on the reflection spectra (c.f. Fig. 3(b)). In this case, the rebar cell concentrates E-field in the area of the rebar's localization and is scattered by the rebars, i.e., the rebar cell affects propagation of microwaves through the specimen. Similar effects of the concentration and scattering of E-field by the rebar cell can be seen in Fig. 4(c) at $2.45 \mathrm{GHz}$ where the resonant peaks can be observed with and without rebars with a very small difference (c.f. Fig. 3(b)). In addition, standing wave patterns inside the specimen can be clearly seen in Fig. 4(c) as a result of the reflection of microwaves from the bottom of the specimen, and decreasing E-field intensity beyond the bottom; that is propagation of microwaves through the specimen is reduced, due to the influence of the rebar cell. Finally, the consideration of E-field distributions at $3.3 \mathrm{GHz}$ shows that the concentrations of E-field in the specimen with 
rebar cell increases, and this can be attributed to resonant conditions in the areas between the rebar cell and the top as well as the bottom of the specimen. The $S_{11}$ is significantly changed at this frequency (i.e., 3.3 GHz) by introducing the rebar cell as can be seen in Fig. 3(b).

\section{TRANSMISSION PROPERTY OF REINFORCED CONCRETE SPECIMENS}

To investigate transmission of microwaves in the reinforced concrete specimen, a microstrip patch antenna was embedded in the specimen. Fig. 5(a) shows a model of the two-antenna setup in CST with MAVA as an external antenna and a microstrip patch antenna as an internal (embedded) antenna operating at $2.45 \mathrm{GHz}$ (referred to as the two-antenna system). The microstrip patch antenna consists of a copper patch and a substrate with dimensions of $35 \times 29 \times 0.035 \mathrm{~mm}^{3}$ and $50 \times 50 \times 1.5 \mathrm{~mm}^{3}$, respectively. The substrate is made of FR4 with relative dielectric constant of 4.3 and loss tangent of 0.025 . Each side of the patch antenna was covered by a foam sheet (as a superstrate) with dimensions of $55 \times 55 \times 10 \mathrm{~mm}^{3}$ and was located in an air box $\left(55 \times 55 \times 23 \mathrm{~mm}^{3}\right)$ inside the specimen at $d_{2}=132 \mathrm{~mm}$ under the center of the rebar cell as shown in Fig. 5(a). Measurement setup included the reinforced concrete specimen and arrangement of the MAVA previous section. In addition, two air-filled grooves were made on the surface of the cube to locate the microstrip patch antenna with a superstrate and a cable connecting the antenna and the PNA as shown in Fig. 5(b).

Then, the cube with the antenna and cable was covered by the rebar cell, plasterboard pieces and concrete slab as described above.

In practice, a typical value of parameter $g$ is in the range of $101.6 \mathrm{~mm}$ to $304.8 \mathrm{~mm}$ [7]. The simulated $S_{22}$ of the patch antenna in free space, concrete and reinforced concrete specimens at $d_{1}=50 \mathrm{~mm}$ and $g=110 \mathrm{~mm}$ is shown in Fig. 6(a). It can be seen from Fig. 6(a) that embedding the patch antenna inside the concrete specimen leads to an increase (relative to free space) of the 
resonant frequency and $S_{22}$ of the antenna. These changes can be attributed to the influence of concrete in E-field of the embedded patch antenna since thickness of the superstrate $(10 \mathrm{~mm})$ and air box $(23 \mathrm{~mm})$ were selected to be relatively small to avoid destruction of concrete integrity. Then, applying the rebar cell in concrete does not change the resonant frequency and $S_{22}$ of the antenna. However, the measured results shown in Fig. 6(b) demonstrate lower shift of the resonant frequency under the influence of concrete than simulated shift, and the influence of the rebar cell on both the resonant frequency and $S_{22}$ can be observed in Fig. 6(b). This discrepancy between simulated and measured results can be explained by the antenna fabrication errors and/or the influence of concrete and the rebar cell on radiation from a connector which is attached to the antenna and connected to the cable.

Fig. 7 shows simulated and measured transmission as $S_{21}$ between two antennas in free space, and with concrete and reinforced concrete specimens at three distances of $d_{1}$. It can be seen from Fig. 7 that the maximum value of the simulated $S_{21}$ in free space is $-15 \mathrm{~dB}$ at $\sim 2.45 \mathrm{GHz}$ and that embedding patch antenna in concrete and applying the rebar cell do not change it at $d_{1}=0$. The maximum $S_{21}$ and corresponding frequency slightly decreases and increases, respectively, at $d_{1}=$ $50 \mathrm{~mm}$ and a notable decrease of the magnitude is observed at $d_{1}=100 \mathrm{~mm}$. Fig. 7 also shows that the changes of $S_{21}$ in the concrete and reinforced concrete specimens are almost the same. This observation leads to the conclusion that the influence of concrete boundaries, in particular the top surface of the specimens is the main contributing factor to changes in the transmission property of the specimens. The measured results shown in Fig. 7 confirm the main features and behavior of the maximum $S_{21}$ which has been observed from the simulated results. On the other hand, the changes in the measured frequency, corresponding to the maximum $S_{21}$ in the specimens (relative to free space case), are negligible, as are the changes of the measured 
frequency corresponding to the $S_{22}$ as shown in Fig. 6(b). It should be noted that the $S_{21}$ is lower in the measurement than in the simulation (c.f. Fig. 7) and this can be attributed to losses in the cable and coaxial connector which were not taken into account in the simulation.

The influence of variation of parameter $\mathrm{g}$ of the rebar cell on $S_{21}$ was investigated numerically at the range from $90 \mathrm{~mm}$ to $170 \mathrm{~mm}$ and the results are shown in Fig. 8(a-c). It can be observed from Fig. $8(\mathrm{a}-\mathrm{c})$ that the decrease of $g$ from $170 \mathrm{~mm}$ to $150 \mathrm{~mm}$ decreases the $S_{21}$, then it increases gradually when $\mathrm{g}$ decreases from $150 \mathrm{~mm}$ to $90 \mathrm{~mm}$, reaching the maximum value at $g=90 \mathrm{~mm}$ which, as expected, is equal to the simulated maximum $S_{21}$ shown in Fig. 7(b). The measurements were conducted with practical values of $g=90 \mathrm{~mm}$ and $110 \mathrm{~mm}$ at three values of $d_{1}$ as shown in Fig. 8(d). It can be seen from Fig. 8(d) that the influence of the change of $\mathrm{g}$ from $90 \mathrm{~mm}$ to $110 \mathrm{~mm}$ on the maximum $S_{21}$ is negligible. The results also demonstrate that the measured maximum $S_{21}$ in Fig. 8(d) is less than the simulated one in Fig. 8(a-c) at $g=90 \mathrm{~mm}$ and the maximum $S_{21}$ for each $d_{1}$ in Fig. 8(d) corresponds to the measured results shown in Fig. 7.

Compared to the results obtained with two $2.5-\mathrm{GHz}$ microstrip patch antennas (one of them was embedded in a concrete cubic specimen similar to that used in this investigation) [6], the increase of $\sim 5 \mathrm{~dB}$ and $\sim 10 \mathrm{~dB}$ is achieved for simulated and measured $S_{21}$, respectively, with the MAVA at $d_{1}=50 \mathrm{~mm}$.

Fig. 9 shows the simulated and measured $S_{21}$ of the two-antenna system with the reinforced concrete specimen possessing the rebar cell when an upper layer of rebars is parallel (case 1) or vertical (case 2) to the E-field polarization vector of the antennas at $d_{1}=0$ and $50 \mathrm{~mm}$. The model for case 1 is shown in Fig. 5(a). It can be seen from Fig. 9 that there is no difference between maximum values of $S_{21}$ obtained at case 1 and case 2 for both the simulated and 
measured results.

\section{CONCLUSION}

The reflection and transmission of microwaves at frequency range of $0.65-3.5 \mathrm{GHz}$ in concrete specimens irradiated by the modified antipodal Vivaldi antenna were investigated numerically and experimentally. It is shown that the reflection from the top of the specimens with and without rebars provided the most critical effect on reduction of transmission in concrete. The investigation into the influence of rebars show that the rebar cell can act as a shield for microwaves if mesh period parameter is less than the electrical half wavelength. The results are used for the investigating the transmission of microwaves at the single frequency of $2.45 \mathrm{GHz}$ between the MAVA and a microstrip patch antenna embedded inside reinforced concrete samples at the centre of the rebar cell. As a future work, the results of this investigation will be used to wirelessly powering the embedded sensors inside concrete and reinforced concrete structures.

\section{REFERENCES}

[1] E. Richalot, M. Bonilla, M.F. Wong, V. Fouad Hanna, H. Baudrand, and J. Wiart, Electromagnetic propagation into reinforced-concrete walls, IEEE Trans. Microw. Theory Techn 48 (2000), 357-366.

[2] R. Paknys, Reflection and transmission by reinforced concrete--numerical and asymptotic analysis, IEEE Trans. Antennas Propag 51 (2003), 2852-2861.

[3] M. Moosazadeh and S Kharkovsky, Development of the antipodal Vivaldi antenna for detection of cracks inside concrete members, Microw Opt Technol Lett 57 (2015), 1573-1578.

[4] R. Zoughi, Microwave non-destructive testing and evaluation, Kluwer Academic Publishers, Dordrecht, Netherlands, 2000.

[5] S. Jiang and S.V. Georgakopoulos, Optimum wireless powering of sensors embedded in concrete, IEEE Tran. Antennas Propag 60 (2012), 1106-1113.

[6] R. Salama and S. Kharkovsky, An embeddable microwave patch antenna module for civil engineering applications, IEEE Int. Instr. Meas. Tech. Conf. (I2MTC), 2013, 27-30.

[7] X. Jin and M. Ali, Reflection and transmission properties of embedded dipoles and PIFAs inside concrete at $915 \mathrm{MHz}$, in Proc. IEEE AP-S Intel. Symp., 2009, 1-4.

[8] G. Ruvio, D. Gaetano, M.J. Ammann, and P. McEvoy, Antipodal Vivaldi antenna for water pipe sensor and telemetry, Int. J. Geophysics 2012 (2012), 1-8. 
[9] F. Fioranelli, S. Salous, I. Ndip, and X. Raimundo, Through the wall detection with gated FMCW signals using optimized patch like and Vivaldi antennas, IEEE Trans. Antennas Propag 63 (2015), 1106-1117.

[10] M. Moosazadeh, S. Kharkovsky, J.T. Case, and B. Samali, Miniaturized UWB antipodal Vivaldi antenna and its application for detection of void inside concrete specimens, IEEE Antennas Wirel. Propag. Lett 16 (2017), 1317-1320.

[11] M. Moosazadeh, S. Kharkovsky, J.T. Case, and B. Samali, Antipodal Vivaldi antenna with improved radiation characteristics for civil engineering applications, IET, Microw. Antennas Propag 11 (2017) 796-803.

[12] M. Moosazadeh and S. Kharkovsky, Design of ultra-wideband antipodal Vivaldi antenna for microwave imaging applications, IEEE Int. Conf. Ubiquitous Wireless Broadband (ICUWB), 2015, 1- 4.

[13] M. Moosazadeh, S. Kharkovsky, J.T. Case, and B. Samali, UWB antipodal Vivaldi antenna for microwave imaging of construction materials and structures, Microw. Opt. Technol. Lett 59 (2017) 1259- 1264.

[14] M. Moosazadeh, S. Kharkovsky, J.T. Case, and B. Samali, Improved radiation characteristics of small antipodal Vivaldi antenna for microwave and millimeter wave imaging applications, IEEE Antennas Wirel. Propag. Lett 16 (2017), 1961-1964.

[15] Z. Esmati and S. Kharkovsky, Modified antipodal Vivaldi antenna for infrastructure health monitoring techniques, IEEE Int. Conf. Ubiquitous Wireless Broadband (ICUWB), 2015, 1-5.

[16] J.Y. Siddiqui, Y.M.M. Antar, A.P. Freundorfer, E.C. Smith, G.A. Morin, and T. Thayaparan, Design of an ultrawideband antipodal tapered slot antenna using elliptical strip conductors, IEEE Antennas Wirel. Propag. Lett 10 (2011), 251-254.

[17] M. Moosazadeh and S. Kharkovsky, A compact high-gain and front-to-back ratio elliptically tapered antipodal Vivaldi antenna with trapezoid-shaped dielectric lens, IEEE Antennas Wirel. Propag. Lett 15 (2016), 552-555. 


\section{University Library}

\section{- M M I N E R VA A gateway to Melbourne's research publications}

Minerva Access is the Institutional Repository of The University of Melbourne

Author/s:

Esmati, Z;Moosazadeh, M

Title:

Reflection and transmission of microwaves in reinforced concrete specimens irradiated by modified antipodal Vivaldi antenna

Date:

2018-09-01

Citation:

Esmati, Z. \& Moosazadeh, M. (2018). Reflection and transmission of microwaves in reinforced concrete specimens irradiated by modified antipodal Vivaldi antenna. Microwave and Optical Technology Letters, 60 (9), pp.2113-2121. https://doi.org/10.1002/mop.31307.

Persistent Link:

http://hdl.handle.net/11343/285089 